\title{
Von der Inkorporierung und Verkörperung des Sozialen zur Somatisierung der Umwelt: Posthumanistische Überlegungen zum biosozialen Subjekt
}

\author{
Anke Strüver \\ Institut für Geographie und Raumforschung, Karl-Franzens-Universität Graz, 8010 Graz, Austria \\ Correspondence: Anke Strüver (anke.struever@uni-graz.at)
}

Received: 19 January 2019 - Revised: 5 May 2019 - Accepted: 27 May 2019 - Published: 25 June 2019

\begin{abstract}
Kurzfassung. With the recognition of relational thinking, methodologies approaching spaces as containers were discarded and reconfigured in social geography. However, there are other "containers" still present in these debates - and present as part of the relations in space - such as the "black box" of the human body. In order to advance the opening of the Black Box of the Body (Guthman, 2012), the article discusses social geographical thinking concerning materiality after the Cultural Turn, which concentrates on a post-dualist and thus a posthumanist concept of materiality.

The ensuing extension of the epistemological critique of dualistic thinking as part of the cultural turn by an ontological critique refers to radical-relational conceptions of NatureCultures (Haraway) and Posthumanist Performativities (Barad), which comprise discursive as well as material power relations. These conceptions neither romanticize nature (or even interpret it in a reactionary way) nor inflate culture. Rather, such a perspective allows to examine the materiality and intra-activity of social environmental conditions at the micro-level of the embodied subject, considering that matter itself acts performatively and even how such performances are enacted.

This article concentrates on theoretical crises within social geography in order to discuss the geography of social crises using the example of environmental injustice and the somatization of the environment with respect to food and health. By extending the "surface"-view on embodied subjects as being socio-culturally encoded and discursively normalized, chemical-biological metabolic processes are also addressed. Therefore, the body is understood as the place where social crises, structures of inequality and discursive categories materialize.
\end{abstract}

\begin{abstract}
„Ways of living and dying matter: ... we must give the best answers we come to know how to articulate, and take action, without the god trick of self-certainty." (Haraway, 2008:88)
\end{abstract}

\section{Einleitung}

Dieser Beitrag nimmt die Aufforderung Donna Haraways, dem Leben (und Sterben) mit mehr Sorgfalt und Umsicht zu begegnen, zum Ausgangspunkt für Ausführungen zur Materialität in der Sozialgeographie. Im Zentrum stehen konzeptionelle Überlegungen zur Inkorporierung gesellschaftlicher Umweltverhältnisse auf der Mikroebene des verkörperten Subjekts. Diese Ausführungen verfolgen das Ziel, Geographien sozialer Krisen und Krisen der Sozialgeographie zusammen zu adressieren (siehe Runkel und Everts, 2017). Diese beiden Krisenphänomene werden natürlich eher selten zusammen gedacht, zumal Erstere weitaus gesellschaftsrelevanter sind als Letztere. Mit dem von mir gesetzten Schwerpunkt, dem verkörperten Subjekt, lassen sich gleichwohl „klassische“ sozialgeographische Themen gesellschaftlicher Ungleichheiten (Rassismus, Sexismus, Klassenfragen), aber auch neuere in Bezug auf Gesundheit, Krankheit, Obdach- oder Wohnungslosigkeit, Sorgebeziehungen, entlang von Aspekten des Alters, der Sexualisierung und Rassifizierung oder Ernährungs- und Umwelt(un)gerechtigkeiten uvm. als ein Ausdruck von multiplen sozialen Krisen aus den Krisen der Sozialgeographie heraus (z.B. Körpervergessenheit, Androzentrismus, biologische Naturalisierungen und kulturelle Essentialisierungen) neu thematisieren. 
„Ob und wie Materialität erfasst werden soll, ist eine der wichtigsten konzeptionellen Fragen der Sozialgeographie" (Becker und Otto, 2016:221). Mit diesem Satz leiten Antje Otto und Sören Becker ihren lesenswerten Einleitungsbeitrag zu aktuellen Herausforderungen der Politischen Ökologie ein und plädieren für eine „konsequente[re] Verknüpfung von ökologischen Fragestellungen mit gesellschaftlichen Verhältnissen“" (ebd., 222), u.a. durch die Aufösung der unterschiedlichen Verständnisse von Natürlichem und Sozialem. Ich werde dieses Plädoyer aufgreifen, sozialgeographisch fortführen ${ }^{1}$ und ein verändertes ontologisches Verständnis von Natürlichem skizzieren, das zwangsläufig auch das Verständnis von Sozialem durchdringt. Mit diesem Ziel vor Augen und dem gewählten Fokus auf den menschlichen Körper wird es gleichwohl nicht um eine Genealogie der Bedeutung der Körperlichkeit in der Sozialgeographie gehen. Ohnehin hat die Körperlichkeit des Subjekts in der deutschsprachigen Sozialgeographie trotz vielerlei begrifflicher Referenzen kaum eine zentrale Rolle gespielt (für komprimierte Übersichten, siehe Weichhart, 2018; Werlen, 2008; für Ausnahmen, siehe Wastl-Walter, 2010 sowie Bauriedl et al., 2010 stellvertretend für feministischgeographische Diskussionen). Unter Umständen wäre noch die leib- und sozialphänomenologische Tradition erwähnenswert, die sich auf das leibliche Erleben konzentriert und die zumeist in explizierter Abgrenzung zur Vorstellung einer „Aktivität von Materialität“ argumentiert ${ }^{2}$, die wiederum für die nachfolgende Beschäftigung mit Geographien sozialer Krisen und Krisen der Sozialgeographie explizit berücksichtigt werden soll. Dementsprechend werden also anstelle der herkömmlichen, oftmals selbstvergewissernden Form von Genealogie zwei der einflussreichsten Autorinnen des New Materialism als onto-epistemo-logischer Denkhorizont etwas ausführlicher vorgestellt und mit Blick auf die Aktivität von Materialität diskutiert, nämlich Donna Haraway und ihre radikal-relationale Konzeption von NatureCultures $(1995,2000,2008,2016)$ und Karan Barads Verständ-

\footnotetext{
${ }^{1}$ Bis auf den hier zitierten Einleitungssatz enthält der Beitrag zur Politischen Ökologie keine weiteren (disziplinpolitischen oder inhaltlichen) Ausführungen zur Sozialgeographie - sehr wohl aber spannende Ausführungen zur Materialität. Von daher verstehe ich ihn auch als Aufforderung, die in dem Aufsatz zur Politischen Ökologie angestoßenen Debatten - v.a. zu gesellschaftlichen Naturverhältnissen - in diesem Themenheft zur Sozialgeographie fortzuführen, u.a. als Auseinandersetzung mit der Inkorporierung gesellschaftlicher Umweltverhältnisse.

${ }^{2}$ Kazig und Weichhart (2009:112f.); so wird bspw. aus Sicht der gegenwärtigen Sozialtheorien die Vorstellung einer Aktivität von Materialität unabhängig von menschlichen Bedeutungszuschreibungen als ,absurd“ abgetan (ebd., 114). Insgesamt scheinen in dieser Denktradition durch den Fokus auf das leibliche erfahrende Subjekt genau die Aspekte verkörperter Subjekte unberücksichtigt zu bleiben, die sich jenseits des individuellen Erlebens durch sozialräumliche Strukturen und gesellschaftliche Diskurse materialisieren (Dörfler und Rothfuß, 2018; siehe auch Hasse, 2017).
}

nis von Posthumanist Performativities (2003, 2007, 2012, 2015). Beide Autorinnen stammen aus dem Kontext der feministischen Naturwissenschaften bzw. Naturwissenschaftsund Herrschaftskritik und haben zentrale Grundlagen für den sich zunehmend etablierenden Ansatz des New Materialism geliefert, der sich mit der Materialität gesellschaftlicher Natur- und Umweltverhältnisse auseinandersetzt und dafür stofflich-materielle Prozesse in sozial- und kulturwissenschaftliche Analysen einbezieht - ohne Materialität zu reessentialisieren oder diskursiv aufzulösen.

Die Perspektive der Feminist Materialisms betont die Notwendigkeit einer kritischer Erweiterung des Cultural Turns und unterstreicht, dass menschliche Körper und Subjekte nicht nur durch gesellschaftliche Interaktionen, Strukturen und Diskurse, individuelle Wahrnehmungen und Praktiken konstituiert werden, sondern auch durch materielle Prozesse, durch Intra-Aktionen bzw. „Verschränkungen“ zwischen Biologischem und Sozialem (Barad, 2015; siehe auch Barad, 2007 sowie einführend Alaimo and Hekman, 2008; Marquardt und Strüver, 2018). Die Ansätze beider Autorinnen eint zudem das Anliegen, Denaturalisierungs- mit Dekulturalisierungsstrategien zusammen zu bringen, um jenseits von biologischen Determinismen und kulturalistischen Essentialismen die Intra-aktivität zwischen Diskursivem und Materiellem als transkörperliche Relationen - als biosoziale Verkörperung - offen zu legen (Abschnitt 3). Dem vorangestellt ist ein kurzer Abschnitt (2) zur geographischen „Black Box of the Body" (Guthman, 2012), der auf Grundlage des relationalen Denkens die Wechselwirkungen zwischen Gesellschaft und Raum am Thema Umwelt(un)gerechtigkeit und damit als Wechselwirkungen zwischen menschlichen Körpern und physischer Umwelt expliziert. Der vierte Abschnitt greift dies als Thema der Sozialgeographie am Beispiel Gesundheit und Ernährung wieder auf; anschließend (5) werden entlang von umweltepigenetischen Aspekten soziale und ökologische Umweltungerechtigkeiten thematisiert. Die Synthese (6) führt die konzeptionellen Abschnitte zusammen und diskutiert, inwiefern sich aus der Krise der Sozialgeographie progressive Ansätze zum Umgang mit Geographien sozialer Krisen ableiten lassen.

\section{Zur Inkorporierung und Verkörperung des Sozialen}

Die für die Sozialgeographie im Zentrum stehenden Wechselbeziehungen zwischen Gesellschaftlichem und Räumlichem lassen sich differenziert nach Skalierungen betrachten (global, national etc., aber v.a. lokal und mikrosozial, wie zwischen Körper und räumlicher Umgebung) sowie differenziert nach konzeptionellen Ansätzen. Die konzeptionellen Ansätze wiederum sind in der Regel entlang verschiedener Epistemologien platziert, sollen hier aber um die onto- 
logische Dimension erweitert werden. ${ }^{3}$ Ausgehend von Elspeth Probyns Auseinandersetzung mit dem ,,spatial imperative of subjectivity“ (2003), Überlegungen zum Cultural Turn, die die Inkorporierung und Verkörperung des Gesellschaftlichen auf der Mikroebene des Subjekts - und des Räumlichen als Teil des Gesellschaftlichen - prominent gemacht haben, rücken mittlerweile neben soziokulturellen Prozessen wie diskursiven Einschreibungen sozialräumlicher Prozesse (Foucault, 1977), normativen Anrufungen und ihren performativen Materialisierungen (Butler, 1993, 1998, 2009) verstärkt Aspekte des materiellen bzw. materialisierten Lebens in den Fokus. Das verkörperte, biosoziale Subjekt ist dann mehr als passives Material, in das sich bspw. rassistische oder sexistische Anrufungen und Diskurse rassifizierend und vergeschlechtlichend einschreiben: Der Körper des Subjekts ist auch der Ort, an dem sich soziale, räumliche und ökologische Ungerechtigkeiten stofflich materialisieren. In diesem Verständnis verändern sich die Elemente und auch der Horizont der Wechselwirkungen zwischen Gesellschaftlichem und Räumlichem, da Natur (hier Umwelt im klassischen Sinne, als materielles Substrat, das mit Lebewesen in einer Wechselbeziehung steht) und körperliche Materie (hier fokussiert auf menschliche Subjekte) wechsel-wirken und u.a. ontologische Erweiterungen zu Fragen des Seins, aber darauf aufbauend auch zu Konzepten von Gerechtigkeit erforderlich werden lassen: Natur ist dann, ,an agentic force that interacts with and changes the other elements in the mix, including the human" (Alaimo and Hekman, 2008:7).

Am Beispiel Umweltgerechtigkeit wird die Materialität des Körpers mit einer kombinierten Perspektive aus Poststrukturalismus und Posthumanismus für die Bearbeitung von sozialgeographischen Forschungsfragen zu bspw. Gesundheit oder Ernährung relevant. Beide Aspekte des Alltagslebens verweisen neben der strukturellen und soziokulturellen Verkörperung auf die stofflich-materielle Inkorporierung von Umgebung (Raumstrukturen) und Umwelt (materielles Substrat, das in Wechselwirkung mit dem Menschen steht) und machen deutlich, dass körperliche Materie kein passives „Ding“ ist, das auf performative Materialisierung durch normative Anrufungen oder diskursive Einschreibungen ,wartet“.

So wird bspw. schlechte körperliche Gesundheit oftmals an Übergewicht festgemacht und dementsprechend wird nach den Ursachen für Übergewicht gesucht: Dominante Erklärungen umfassen zum einen strukturbasierte Gleichsetzungen wie „dick, doof und arm“ (kritisch: Schorb, 2009) sowie die an Angebot und Nachfrage orientierten politökonomischen Verhältnisse (Guthman and DuPuis, 2006), wobei

\footnotetext{
${ }^{3}$ Dies schließt auch die Kritik an der Essentialisierung von Skalierungen mit ein (siehe die durch Sallie Marston u.a. angestoßene Debatte, vgl. Marston, 2000; Marston et al., 2005; Jones III et al., 2007); diese bleibt im Folgenden aber unberücksichtigt; ebenso unberücksichtigt bleiben in diesem Beitrag die Debatten um NonRepresentational Theory (vgl. Thrift, 2007).
}

ein Zusammenhang zwischen Übergewicht und niedrigem sozioökonomischen Status, bestimmt durch Einkommensund Bildungsniveau, hergestellt wird. ${ }^{4}$ Dem gegenüber steht zum anderen ein Fokus auf individuelles (Fehl-)Verhalten, der diskursive Körpernormen und normalisierte Bewegungsformen manifestiert und dabei ebenfalls auf die normativpolitische Gleichsetzung von dick = ungesund rekurriert (zum Verhältnis von fat blaming und fat shaming, siehe Schorb, 2015) bzw. an die Selbstkontrolle i.S.e. moralischen Selbstführungsimperativs appelliert. Als neuerer Zugang jenseits der Polarisierung „Verhältnisse oder Verhalten“ wird hier der biophysische bzw. biosoziale Ansatz aus dem Kontext des New Materialism eingeführt, eine ,political ecology of the body“ (Guthman, 2012), der sich auf die „Natur“ der physischen Umwelt und auf die „Natur“ der menschlichen Körper konzentriert (s.u.).

Gesellschafts- und Wissenschaftskritische Forschungen aus dem Bereich der Gesundheits- und Umweltgerechtigkeit dehnen aktuell strukturalistische und poststrukturalistische Körperkonzeptionen aus, um stofflich-materielle Prozesse und biologische Effekte gesellschaftlicher Ungleichheiten wie Rassismus oder Sexismus einzubeziehen (siehe bspw. Pulido, 2016). Fragen nach Gerechtigkeit lassen sich mit unterschiedlichen Ansätzen diskutieren, die u.a. danach differenziert werden, wie sie Entstehung und Manifestation von Ungerechtigkeit erklären. Iris Marion Young beispielsweise unterscheidet Formen von struktureller Marginalisierung und kulturellem Imperialismus (Young, 2011) die gleichwohl beide in Ungleichheiten und Ungerechtigkeiten münden. Nancy Fraser (2009) geht es hingegen viel stärker um die Anerkennung von struktureller Ungleichheit, um durch ökonomische Umverteilung und politische Beteiligung Ungerechtigkeit zu bekämpfen - wobei Umverteilung nicht Gleichheit, Gleichbehandlung oder Gleichverteilung entspricht, sondern i.S. einer erweiterten Verteilungsgerechtigkeit zu verstehen ist. Neben den klassischen Beispielen aus dem globalen Nord-Süd-Kontext eignet sich für sozialgeographische Kontexte die lokale Ebene als Ansatzpunkt, um Ungerechtigkeit zu erfassen und zu bekämpfen. Dabei geht es weder um die Romantisierung noch Universalisierung des Lokalen, sondern um die Wechselwirkungen zwischen globalen Krisen (wie ökologischer oder ökonomischer Krise) und lokalem Leben. Der Rekurs auf die biologische Materialität gesellschaftlicher Körperphänomene basiert dabei nicht auf einem Verständnis von biologischen Grundlagen von Rassismus (oder Sexismus). Im Fokus ste-

\footnotetext{
${ }^{4}$ Der essende Körper kann in dieser Rahmung gar zum ,spatial fix“ für kapitalistische Probleme werden: „Fast food becomes a double good fix for capitalism: not only does it involve the superexploitation of the labor force, it also provides an outlet for surplus food. Insofar as this surplus manifests in obesity, the contradiction is (temporarily) resolved in the body. (...) Neoliberalism's other fix is to create purchasable solutions to the problem it generates. One solution ... is to commodify dieting as well as eating " (Guthman and DuPuis, 2006:441; siehe auch Guthman, 2012, 2015).
} 
hen vielmehr die biophysiologischen, stofflichen Effekte gesellschaftlicher Ungleichheitsstrukturen: „Environmental justice movements epitomize a trans-corporeal materiality, as conception of the body that is neither essentialist, nor genetically determined, nor firmly bounded, but rather a body in which social power and material/geographic agencies intraact" (Alaimo, 2010:63).

\section{Zur Aktivität von Materialität: Transkörperliche Relationen}

Das vermeintliche „Warten“ der körperlichen Materie auf Bedeutungszuweisungen - durch gesellschaftliche Interaktionen, diskursive Einschreibungen und deren performative Materialisierungen - hat Haraway bereits in den 1980er Jahren als ein reduktionistisches Verständnis von verkörperten Subjekten (bzw. von materieller Stofflichkeit im Allgemeinen) kritisiert, denn der Körper ist mehr ,,als ein leeres Blatt für soziale Einschreibungen, einschließlich derjenigen des biologischen Diskurses“ (Haraway, 1995:92). ${ }^{5}$ Sie bezieht sich damit auf die unauflösbaren Verschränkungen zwischen Materialität und Repräsentation (als materiell-semiotische) sowie zwischen Natur und Kultur (als NaturKultur) und argumentiert gleichzeitig de- und re-ontologisierend. Ihre radikal-relationale Konzeption widerlegt die klare Abgrenzung zwischen Materiell-Stofflichem und DiskursivRepräsentationalem - und sie widerlegt sowohl einen biologischen Determinismus (,Natur") als auch einen rein sozialen Konstruktivismus (,Kultur“). Ihre Konzeption der NaturKultur erfasst den Körper als einen materiell-semiotischen Prozess, in dem Materiell-Stoffliches und SprachlichKulturelles untrennbar verschränkt sind bzw. in dieser Verschränkung miteinander interagieren (,response-abilities“, Haraway, 2016:115): „Eine biologische Zelle ist unser Name für Prozesse, die nicht abgrenzbar und unabhängig von unseren Interaktionen sind.“ (Haraway, 2000:24f., Übers. A.S.).

Haraways Fokus auf die Aktivität der Materialität und ihr Konzept des Körpers als materiell-semiotischer Prozess wird von Barads Verständnis von Materie als Agens weiterentwickelt: Ihr Programm des Agentiellen Realismus - dem Vorstoß, Konstruktivismus und Realismus nicht als Widerspruch zu denken $(2003,2007,2012,2015)$ - schlägt einen posthumanistisch performativen Ansatz natürlich-kultureller Praktiken vor, der die dynamische Kraft der Materie ernst nimmt. Materie ist dann nicht länger Ding(-lich), sondern Agens, das sich als Teil intra-aktiver Prozesse materialisiert. Barad radikalisiert Haraway, indem sie „Materie [als] Substanz in ihrem intra-aktiven Werden - kein Ding, sondern eine Tätigkeit, eine Gerinnung von Tätigsein“ (Barad, 2012:40) fasst; und sie radikalisiert Butler, indem sie Performativität nicht

\footnotetext{
${ }^{5}$ Mit ähnlichem Wortlaut betont auch Barad (2015:61): „Natur ist weder ein leeres Blatt für das freie Spiel sozialer Einschreibungen, noch irgendeine vermittelt vorhandene, transparent gegebene ,Dingheit' [thingness].“
}

auf die Wiederholungen sprachlich-diskursiver Anrufungen reduziert, sondern um intra-aktive Wiederholungen erweitert. D.h. Butlers Argument der performativen Materialisierung von verkörperten Subjekten ausbauend betont Barad die dynamische Intra-Aktivität zwischen Materiellem und Diskursivem in verkörperten Subjekten; und sie betont die Intraaktionen zwischen Bedeutung und Materie, ,,von Wissen und Sein, Wort und Welt, Kultur und Natur. Phänomene sind materiell-kulturelle Da-Seins" (Barad, 2015:61; siehe auch Frost, 2016). Diese Intra-aktionen umfassen Agieren jenseits des Sprachlichen und Menschlichen, so dass Barad von posthumanistischer Performativität spricht. Mit ihrem Konzept der „Onto-Epistemo-logie“ (2012) lehnt sie einen einseitigen Repräsentationalismus ab, d.h. die Vorstellung, dass die Bedeutungen der Objekte der sozialen Welt ausschließlich durch menschliches Agieren konstituiert werden, dass also wie im klassischen dualistischen Denken - nur Subjekte aktiv und die Objekte passiv sind. ${ }^{6}$ Um diesen Dualismus zu überkommen, um die unterschiedlichen ontologischen Verständnisse von Natürlichem und Sozialem aufzuspüren und um Materie zu (re-)aktivieren, bedarf es einer posthumanistischen Auflösung des Natur-Kultur-Dualismus, die Barad (2007, 2012) folgendermaßen skizziert: Anstelle eines relationalen, aber dualistischen Denkens, in dem die Materie nur in Relation und Interaktion mit dem Menschen Bedeutung erhält, spricht sie von transkörperlichen Relationen in Intra-Aktion. Das Programm des Agentiellen Realismus basiert auf räumlich lokalen und mikrosozialen verkörperten Erfahrungen, die in der ontologischen Untrennbarkeit von Materiellem und Kulturellem gemacht werden - und für die epistemologische wie ethische Verantwortung übernommen werden muss (Barad, 2015:43ff.).

Verschiedene feministische Ansätze aus den Natur-, Sozial-, Kultur- und Geisteswissenschaften sowie soziale Bewegungen aus dem feministisch-ökologischen Kontext haben sich seit langem mit den Verhältnissen von menschlichen Körpern zu Natur und Umwelt beschäftigt und die Zusammenhänge zwischen materiell-körperlichen Lebensbedingungen, sozialer Gerechtigkeit und Umwelt entlang verschiedener Krisen und auf unterschiedlichen Maßstabsebenen problematisiert (siehe bspw. Cağlar et al., 2012). Die feministische Literaturwissenschaftlerin Stacey Alaimo hat u.a. Barads Plädoyer für den Fokus auf transkörperliche Relationen aufgegriffen und spricht in diesem thematischen sowie im posthumanistischen theoretischen Kontext von ,trans-corporeal subjectivity in which bodies extend into places and places deeply affect bodies“ (Alaimo, 2016:5).

\footnotetext{
${ }^{6}$ In den meisten poststrukturalistischen Konzepten wurden ontologische Überlegungen zu den Bedingungen des Seins als Teil des anti-essentialistischen Anspruchs explizit ausgeklammert. So hat bspw. Butler $(1993,1998)$ im Zuge ihrer Konzeption der performativen Materialisierung gefordert, den Körper weniger als Seiendes zu betrachten, denn als Ort der politischen Repräsentation und Regulation.
} 
Und wenn die körperliche Physis des Subjekts mit der Umwelt in einem intraaktiven Verhältnis steht, verändert dies das Verständnis von Sozialem und verkörperter Subjektivität: „As the material self cannot be disentangled from networks that are simultaneously economic, political, cultural, scientific, and substantial, what was once the ostensible bounded human subject finds herself in a swirling landscape of uncertainty where practices and actions that were once not even remotely ethical or political matters suddenly become the very stuff of the crises at hand. This is especially evident in the case of global climate change: an individual, household, business, university, city, state, nation, or continent can calculate the carbon footprint left by the stunning range of human activities that emit carbon. I think it is crucial to emphasize, however, that transcorporeality, as it emerges in environmental health, environmental justice, web-based subcultures, green consumerism, literature, photography, activists websites, and films, is a recognition not just that everything is interconnected but that humans are the very stuff of the emergent, material world“ (Alaimo, 2010:20). Dabei spielt der von Barad eher beiläufig verwendete Hinweis auf transkörperliche Relationen in den empirischen Erläuterungen durch Alaimo (2016) eine zentrale Rolle und wird hier als Rahmung fortgeführt: „I propose the term ,trans-corporeality ', in which human bodies are not only imbricated with one another but also enmeshed with nonhuman creatures and landscape“ (ebd., S. 67). Erweitert werden damit die Wechselwirkungen zwischen Körpern und Raum (iSv Umgebung) um die ökologischen Beziehungen zwischen Lebewesen und Umwelt.

\section{Zur Somatisierung der Umwelt}

Die menschliche Gesundheit - und Ernährung als Teil der Gesundheit - sollen hier als illustrierende Beispiele für transkörperliche Relationen, für die Wechselwirkungen zwischen Gesellschaft und Raum sowie zwischen Mensch und Umwelt als ,Somatisierung von Umwelt“ (Guthman, 2014:1166). ${ }^{7}$ In Erweiterung des biomedizinischen Paradigmas in der Geographie, also der Annahme, dass Gesundheit und Krankheit rein biologische Phänomene sind, um sozialepidemiologische bzw. kultur- und sozialwissenschaftliche Gesundheitskonzepte der Geographies of Health (vgl. Brown et al., 2017; Gatrell and Elliott, 2015), stand v.a. die Absage an biologische Determinismen im Vordergrund, die allerdings mit der Vernachlässigung der stofflichen Materialität des Körpers einherging. D.h. nach den epistemologischen Turns (v.a. im Zuge des Cultural Turn) bedarf es jetzt eines posthumanistischen ontologischen Turns, der transkörperliche Relationen integriert.

\footnotetext{
${ }^{7}$ Erweitert wird damit auch die These von der „Somatisierung des Sozialen“ (Bourdieu, 1992), die die Inkorporierung und Verkörperung sozialer Identitäts- und Strukturkategorien sowie hegemonialer Klassifikationssysteme umschreibt.
}

In der klassischen humangeographischen Betrachtung von Gesundheit geht es v.a. um die Suche nach Kausalitäten zwischen Gesundheit bzw. Krankheit und einem bestimmten Raumausschnitt. Dahinter steht die Annahme, dass ein Raum und seine Ausstattung auf die Menschen ,wirke“ (Wohnverhältnisse, Grün- und Freiräume, Belastungen durch Lärm, Umweltverschmutzung etc.); das verkörperte Subjekt spielt darin nur eine untergeordnete Rolle. Dementsprechend konzentrieren sich aktuell die meisten Forschungen im Globalen Norden auf den Einfluss von gesundheitsrelevanten Infrastrukturen sowie von räumlich situierten Umweltstressoren auf die Bevölkerung (vgl. Oßenbrügge und Strüver, 2018).

Aktuelle Ansätze zur Gesundheits- und Umweltgerechtigkeit und aus der ökosozialen Epidemiologie (Krieger, 2013; siehe auch Bolte, 2018) verweisen hingegen auf Perspektiven, die diesem Problem der einseitigen Betrachtung (sozialräumliche Strukturen beeinflussen die Gesundheit) sowie der ebenfalls einseitigen „Alternative“ (individuelles Verhalten beeinflusst die Gesundheit, s.o.) etwas entgegensetzen: Gesundheit und Krankheit scheinen jenseits von Verhältnissen oder Verhalten auch darauf zurückzugehen, dass die räumliche Umgebung iSv Umwelt im wahrsten Sinne des Wortes in Fleisch und Blut übergeht, d.h. dass Schadstoffe in Luft, Wasser und Böden, Pestizidrückstände in Lebensmitteln und andere Toxine als bioaktive Substanzen im Körper wirken und die Gesundheit beeinflussen. Stressoren aus Umwelt und Lebensmitteln verursachen dabei nicht nur akutes physisches oder psychisches Unwohlsein, sondern können die Entwicklung ganzer Communities beeinflussen (s.u.). Gerade in den USA gibt es unzählige Beispiele für ,,strukturellen Umweltrassismus“ (Pulido, 2016; Walker, 2009), die deutlich machen, dass insbesondere Black Communities von den Folgen von Umweltungerechtigkeit nicht nur soziokulturell, sondern auch biophysisch betroffen sind, da sich Umweltstressoren und ,schlechte“ Ernährung in der biophysikalischen Stofflichkeit des Körpers, z.B. als Fettzellen, materialisieren. ${ }^{8}$

Damit können rein sozial- und kulturwissenschaftliche Perspektiven um biologisch-materielle Prozesse - jenseits biomedizinisch-naturwissenschaftlicher Annahmen ergänzt werden. Dies gilt auch für die körperliche Produktion und die Aktivität von Fettzellen, so dass dominante Erklärungen für Fettleibigkeit (sozialräumliche Verhältnisse oder

\footnotetext{
${ }^{8}$ Dazu gehören die so genannte „Cancer Alley“ der Petrochemie entlang des Mississippi in Louisiana (Zimring, 2016; siehe Hochschild, 2016 für eine Studie zum Umgang der weißen Bevölkerung mit diesem Phänomen), die zahlreichen Mülldeponien im Südosten der USA (Bullard, 2000), aber auch allgemeiner die überdurchschnittliche hohe Rate an lebensbedrohlichen Krankheiten unter Schwarzen Arbeiter*innen bzw. in Black Communities (Alaimo, 2010; Gravlee, 2009; Pulido, 2016; Taylor, 2014). Anhand der Auswirkungen des Hurricane Katrina in New Orleans erläutert zudem Tuana (2008) die „Porosität“ zwischen menschlichen Körpern und Umwelt(-giften), die neben den Folgen von sozioökonomischer und soziokultureller Ungerechtigkeit zu schweren Erkrankungen insbesondere der Schwarzen Bevölkerung geführt hat.
} 
individuelles Verhalten) zu erweitern sind: Fettleibigkeit und Übergewicht können dann nicht länger nur sozialstrukturelle, kulturelle und räumliche Ursachen haben, sondern auch Effekte von Umweltstressoren sein, die sich in transkörperlichen Intra-aktionen, im materiell-kulturellen Da-Sein festsetzen (Guthman, 2013; Kuzawa and Sweet, 2009; Mansfield, 2012). Essen - als Produkt und als Praktik -, körperliche Bewegungen und Körpergewicht erweisen sich damit als Teil sozialer und räumlicher Ungleichheiten und Relationen, aber auch als Intra-aktionen zwischen Biologischem und Sozialem. Fettleibigkeit ist dann nicht nur auf makrosoziale Strukturen, räumliche Verhältnisse und/oder mikrosoziales Verhalten (Bewegung, Ernährung) zurückzuführen, sondern kann auch durch epigenetische Veränderungen bedingt sein. Damit angesprochen wird die Inkorporierung als Somatisierung von biophysischen Umweltverhältnissen als transkörperliche Relationen, einschließlich ihrer biochemischen, wie auch diskursiven und sozialstrukturellen Stressoren. Zu letzteren gehören z.B. rassistische, sexistische oder fat blaming Anrufungen sowie an solche Stigmatisierungen gebundene strukturelle Ungleichheiten und deren Inkorporierung. Die Somatisierung von biophysischer Umwelt verlangt gleichwohl die Auflösung der ontologischen, epistemologischen und stofflichen Grenzen zwischen Mensch und Umwelt, zwischen Sozialem und Natürlichem - und es erfordert bzw. ermöglicht eine posthumanistische Erweiterung der Begriffe „Wechselwirkung“ und „Stoffwechsel“.

Anhand von Essen - als Produkt wie als Praktik und den damit verbundenen Stoffwechselprozessen - lässt sich die stofflich-materielle Inkorporierung und Somatisierung von Raumstrukturen, von Umwelt- und Ungerechtigkeitsverhältnissen beispielhalft illustrieren: Dies schließt die menschliche Aktivität des Essens (auch als Materialität von Aktivität) und die Aktivität des Essens (einschließlich der Aktivität von Materialität) mit ein, da die körperliche Materie nicht passiver Rezipient von Diskursen über Ernährungsformen und Körpernormen ist. Guthman (2014:1155) bringt das folgendermaßen auf den Punkt: „Food enters into bodies and has bodily manifestations - as energy, disease, conditions, morphology. This must be true no matter how influential social and cultural factors are in affecting how and what we eat and what we look like."

In den hier vorgestellten posthumanistischen Überlegungen basieren Essen und essen auf Intra-Aktivitäten und stellen eine ultimative NaturKultur dar, als Lebens-Mittel (human) und Lebens-Mittel (nicht-human). Essen und essen sind beide Bestandteile der Einverleibung gesellschaftlicher Naturverhältnisse in den Verständnissen der politischen Ökonomie und politischen Ökologie, aber auch der Somatisierung biostofflicher Umweltverhältnisse im Sinne des New Materialism, d.h. einschließlich biophysischer und möglicher epigenetischer Folgen. Seit Marx wird der MetabolismusBegriff für die Stoffwechselprozesse zwischen Mensch und Natur verwendet, z.B. für Essen als Produkt, das als Ware aus sozioökonomischen und ökologischen Ausbeutungsver- hältnissen hervorgeht (vgl. Hirth, 2018; siehe auch Guthman, 2015). Ursprünglich verweist Metabolismus als griechisches Wort für „Umwandlung“ gleichwohl auf körperliche Stoffwechselprozesse - auf Prozesse, die durch die Aktivität von Materialität (Essen) und die Materialität von Aktivität (essen) den Körper leben (oder sterben) lassen. Essen und essen und die daran gebundenen Stoffwechselprozesse dynamisieren damit das Verständnis von materieller Stofflichkeit und verweisen auf die Frage, wie menschliche Materie (Körper) und stoffliche Materie (Essen) mit kurz-, mittel- und auch langfristigen Auswirkungen intraagieren. So schreibt bspw. Bennett (2007) Essen eine agentische Fähigkeit zu, die zwar untrennbar von menschlichem Agieren ist, aber keinesfalls auf essen als menschliche Tätigkeit reduziert werden kann (siehe auch Mol, 2008; Landecker, 2011). ${ }^{9}$ Mit Bezug auf den eingangs kurz angesprochenen Aspekt der Verteilungsgerechtigkeit (Fraser, 2009) muss es daher in der Erörterung von Umwelt(un)gerechtigkeit darum gehen, neben ökonomischen und soziokulturellen auch ökologische Ungleichheiten als Ungerechtigkeiten anzuerkennen und soziale Ungerechtigkeit als im verkörperten Subjekt biologisch materialisierte $\mathrm{zu}$ thematisieren.

\section{Zur Erweiterung der Materialität von Aktivität: Umweltepigenetik}

Am Beispiel der These der „Obesogenic Environments “ (vgl. Townshend and Lake, 2017) lässt sich der Stoffwechsel zwischen sozialräumlichen Verhältnissen und verkörperten Subjekten als Bestandteil der dominanten kapitalistischen Logik sowie als Beispiel für ungesunde und ungerechte städtische Lebensformen veranschaulichen. Der Fokus auf Gerechtigkeit erweitert die Perspektive jenseits von Verhältnissen oder Verhalten darauf, dass neben der sozialräumlichen Umgebung auch die biophysische Umwelt somatisiert wird, da Schadstoffe aus Böden, Luft, Lebensmitteln und Wasser als bioaktive Substanzen im Körper wirken. D.h. derartige Umweltstressoren können ernsthafte Krankheiten verursachen; sie können aber auch die phänotypische Entwicklung von Familien langfristig beeinflussen. Guthman (2013) dekonstruiert die These der „dickmachenden Stadtteile“ dahingehend, als dass sie Übergewicht nicht nur auf das Fehlen einer körperliche Aktivitäten begünstigende städtische Infrastruktur (v.a. Rad- und Fußwege) sowie eine schlechte Lebensmittelnahversorgung (mit frischen und unverarbeiteten Waren) reduziert. Zusätzlich zur Kritik an der fehlenden Berücksichtigung der Gründe für die fehlende Wege- und Versorgungsinfrastruktur (mangelnde öffentliche Investitionsbereitschaft, keine Investitionsanreize für Unternehmen) sowie

\footnotetext{
${ }^{9}$ An anderer Stelle wird in diesem Zusammenhang von einem Visceral Turn gesprochen, der sich insbesondere der Aktivität physischer Materialität im essenden Körper widmet (Hayes-Conroy and Hayes Conroy, 2015), für empirische Ansätze dazu, siehe bspw. Sexton et al., 2017; Evans und Miele, 2012; Bruckner, 2018).
} 
der Gründe für die Wahl eines Quartiers (günstige Mietund Immobilienpreise), stellt sie auch die oftmals überdurchschnittlich hohen Umweltbelastungen in solchen Quartieren auf den Prüfstand: Diese halten zwar die Immobilienpreise niedrig, können aber akut wie epigenetisch zur Veränderung von Fettzellen und z.B. zur Entstehung und Vererbung von Übergewicht führen. Und es ist v.a. der letztgenannte Aspekt, der trotz der Unterschiede der nordamerikanischen und europäischen Diskurse die These der Obesogenic Environments aktuell jenseits regionaler Spezifika relevant macht. Neben der grundsätzlichen Hinterfragung der Gleichsetzung von Übergewicht mit Krankheit sowie der fat blaming- und fat shaming-Diskurse und ihren Inkorporierungen wird gefordert, den biosozialen Einflüssen von Umwelt(-stressoren) mehr Beachtung in der Forschung zur lokalen Umweltgerechtigkeit entlang der Alltagsaspekte Ernährung und Gesundheit zu schenken (Guthman, 2013; Evans and Colls, 2014; Schorb, 2015).

Dieses Öffnen der Black Box of the Body erlaubt, die Aktivität von körperlicher Materie wie (Fett-)Zellen und Gewebe zu analysieren und dadurch die dominanten Erklärungen für - und Präventionsmaßnahmen gegen - Fettleibigkeit zu multiplizieren. Die raumbasierten Ursachen für Fettleibigkeit liegen dann nicht länger nur in soziokulturellen Strukturen - sie können auch Effekte von Umweltstressoren sein, die sich in der biophysikalischen Stofflichkeit des Körpers materialisieren. Körperfett und -gewicht können somit als aktiver Teil gesellschaftlicher und räumlicher Ungleichheiten berücksichtigt werden, als transkörperliche Relationen zwischen Biologisch-Natürlichem und Sozial-Kulturellem. D.h. die Ursachen für die Exposition von Umweltstressoren gehen auf sozioökonomische und -kulturelle Ungerechtigkeiten in der segregierten Stadt zurück, doch die Folgen sind sowohl soziale als auch biophysische, da sich urbane sozialräumliche Ungerechtigkeiten stofflich im Körper materialisieren: „Embodiment is a matter not of being specifically situated in the world, but rather of being of the world in its dynamic specificity“ (Barad, 2007:377, Hervorh. i.O.). Biologische Aspekte determinieren damit nicht die verkörperte Subjektivität, doch die Epigenetik zeigt, dass verkörperte Subjekte nicht nur Effekt gesellschaftlicher Verhältnisse und individualisierten Verhaltens sind. „Bodies do not simply take their places in the world. They are not simply situated in, or located in, particular environments. Rather, ,environments' and ,bodies' are intra-actively co-constituted" (ebd., S. 170).

Um die Black Box des Körpers als auch die der Umwelt zu öffnen, um deren radikale Relationalität zu adressieren sowie eine relational-posthumanistische und intra-aktive Ontologie zu praktizieren (i.S.v. Haraway und Barad, s.o.), eignen sich u.a. umweltepigenetische Forschungen. Eingebettet in den Kontext des Feminist New Materialism (z.B. Alaimo and Hekman, 2008; Alaimo, 2010, 2016; Braidotti, 2014; Frost, 2016; Guthman, 2014; Guthman and Mansfield, 2013; Löw et al., 2017; Mansfield and Guthman, 2015; Mansfield, 2012,
2017; Müller, 2017) lassen sich so die Beziehungen zwischen genetischen Veranlagungen, körperlicher Gesundheit und Umwelteinflüssen dynamisieren, da epigenetische Mechanismen die Genexpressionen (nicht aber die Gene selbst) beeinflussen können.

In dieses Verständnis spielt eine dynamische, denaturalisierte biologische Ontologie mit hinein: „What happens inside bodies, even at the molecular level, is entirely dependent on what happens outside of them - including the past. So, by focusing on the ways environments interact with genetics to shape bodily outcomes, epigenetics is inherently spatiotemporal and socionatural. Supporting much contemporary social theory epigenetics blurs dualistic distinctions" (Mansfield and Guthman, 2015:4). In Bezug auf soziale und ökologische Umweltgerechtigkeit bietet die Umweltepigenetik Möglichkeiten offen zu legen, wie sich Umwelteinflüsse im biologischen Körper materialisieren und so biologische Differenzen, z.B. zwischen Menschen verschiedener Hautfarben produzieren; „how race becomes biology“ (Gravlee, 2009:47; Hervorh., A.S.). Damit kann biologisch determiniertem und legitimiertem Rassismus (und auch Sexismus ${ }^{10}$ ) die Grundlage entzogen werden - ohne rein strukturalistisch und/oder kulturalistisch zu argumentieren. „In the emerging science of environmental exposure, in fields such as epigenetics, all environments are understood as chemicals. Ecological, biological political, economic, cultural, social and other dynamics all become biological as chemicals that come from outside the body (that we ingest in food and water, breathe in the air, that cross directly through our skin and placentas), or as chemicals that are made inside the body in reaction to external events“" (Romero et al., 2017:167).

Wenn xenobiotische Umweltgifte im menschlichen Körper intra-agieren und ihn sowie seine Nachkommen phänotypisch verändern, stellen Föten die „Zeugen“ vergangener Umwelteinflüsse und - ungerechtigkeiten dar (Mansfield, 2012, 2017). Dieser Aspekt der Epigenetik beinhaltet gleichwohl auch das Problem einer erneuten Responsibilisierung, der individualisierten Verhaltensverantwortung für die eigene Gesundheit und die der nachkommenden Generationen, da es als moralische Pflicht erscheint, ein gesundes Leben zu führen. Auch wenn das epigenetische Fundament auf einer anderen disziplintheoretischen Logik basiert als Foucaults Biopolitik (2006), so wird gerade durch die Ähnlichkeit des Selbstoptimierungsarguments in beiden Ansätzen deutlich, dass die Epigenetik als Gesellschaftskritik nur funktioniert, wenn sie sozialtheoretisch rückgebunden wird. ${ }^{11}$

\footnotetext{
${ }^{10}$ Zur biologischen Materialisierung von sozialem Geschlecht, siehe Fausto-Sterling (2012).

${ }^{11}$ Denn die Epigenetik als Eugenik liefert durch die Abkehr vom biologischen Determinismus prinzipiell auch Möglichkeiten für Formen der Optimierung des Selbst und der Nachfahren, v.a. durch den Anspruch, die Exposition gegenüber positiven wie negativen Umweltstressoren zu steuern (siehe bspw. Meloni, 2016).
} 
Die Umweltepigenetik verweist auf eine biologische Inkorporierung der materiell-stofflichen Umwelt, nämlich neben der Verkörperung von diskursiven und sozialen Faktoren auch auf die Somatisierung biophysischer und xenobiotischer Stressoren. Erforderlich wird dafür die Auflösung des ontologischen Dualismus von Kultur und Natur und eine posthumanistische Neudefinition von Mensch-Umwelt-Beziehungen als transkörperliche Relationen: „Trans-corporeality is a new materialist and posthuman sense of the human as perpetually interconnected with the flows of substances and the agencies of environments" (Alaimo, 2016:112). Dies beinhaltet einen Fokus auf die Intra-aktionen zwischen soziokulturellen und stofflichen-materiellen Dimensionen, so dass sozialräumliche und raumzeitliche Erfahrungen nicht nur repräsentationell, sondern auch materiell in Fleisch und Blut übergehen (Fausto-Sterling, 2012; Tolia-Kelly, 2013).

Eine Perspektive auf die menschliche Gesundheit als biosozial, als - auch stoffliches - Verhältnis zwischen Natur und Kultur, umfasst ein Verständnis von Natur jenseits biologischer Determinismen - aber auch jenseits kultureller Essentialismen sowie gesellschaftlicher Verhältnisse und individuellem Verhalten; die Gesundheit menschlicher Körper ist dann gleichzeitig biophysisch und sozial (Mansfield, 2008:1015; siehe auch Alaimo 2010, 2016; Braidotti, 2014; Haraway, 2008, 2016), also biosozial. ${ }^{12}$ In dieser radikalrelationalen, posthumanistischen Forschung wiederum wird die klassisch-konstruktivistische sozial- und kulturwissenschaftliche Frage ,wie wird eine biologische Differenz zwischen verkörperten Subjekten sozial wirkmächtig?" erweitert um eine „biosozial-materielle“ Frage, d.h. „wie wird eine soziale Differenz körperlich-biologisch wirkmächtig?“" Diese Erweiterung bzw. Reformulierung berücksichtigt, dass die Gründe für Exposition von Umweltstressoren oft auf sozioökonomische und -kulturelle Ungerechtigkeiten zurückgehen, doch die Folgen auch biophysiologische sind. Eine New Materialism-Perspektive auf biosoziale und biopolitische Prozesse bietet sich somit auch an, um Foucaults in strukturalistischen wie poststrukturalistischen Kontexten dominante Überlegungen zum Biologischen als Teil des Politischen weiterzuführen (Foucault, 1999): denn während Foucault sich dafür interessierte, wie biologische Differenzen als Rassismus (und Sexismus) sozial „praktiziert“ werden, und darlegt, wie sie sich als biopolitische Rationalitäten und gesellschaftliche Regulierungsmechanismen manifestieren eine Rationalität, die zwischen verschiedenen Hautfarben und Geschlechtern differenziert - rücken nun die biophysischen Ungleichheiten als Effekte sozialer Ungleichheiten in den Fokus und erlauben gesellschaftliche Ungerechtigkeiten neu zu problematisieren.

\footnotetext{
${ }^{12}$ Frost (2016) argumentiert sehr ähnlich, bezeichnet den Menschen gleichwohl als biokulturelles Wesen, als Prozessprodukt aus kultureller Umgebung und ökologischer Umwelt.
}

6 Synthese und Ausblick: Biosoziale Verkörperungen und intra-aktive Mensch-Umwelt-Beziehungen

„Further, syntactically and materially, worldly embodiment is always a verb, or at least a gerund. Always in formation, embodiment is ongoing, dynamic, situated, and historical. No matter what the chemical score for the dance - carbon, silicon, or something else - the partners in infoldings of the flesh are heterogeneous. That is, the infolding of others to one another is what makes up the knots we call beings“" (Haraway, 2008:249f.). ${ }^{13}$

„Die aktuelle globale Wirtschaftskrise ist nicht nur eine Krise der Wirtschaft und des Staates, sondern auch eine der Gesellschaft und ihrer verkörperten Subjekte" (Dzudzek und Strüver, 2013:145). Wie lässt sich diese Feststellung, ebenfalls ein Einleitungssatz zu einem Themenheft der Geographica Helvetica, in Verbindung bringen mit, erstens, der ökologischen Krise, zweitens, der eingangs zitierten Aufforderung zur Beschäftigung mit Materialität als ,eine der wichtigsten konzeptionellen Fragen der Sozialgeographie“ (Becker und Otto, 2016:221), drittens, den europaweit beobachtbaren Problemen wie gesellschaftliche Des-Integration und Rechtsruck, zunehmende sozialräumliche Ungerechtigkeiten etc. als Ausdruck von Geographien sozialer Krisen (Runkel und Everts, 2017), und viertens, Haraways Aufforderung to „take action, without the god trick of self-certainty“ (Haraway, 2008:88)?

Eine der - nicht großen, aber lang anhaltenden (und sich voraussichtlich fortsetzenden) - Krisen der deutschsprachigen Sozialgeographie liegt in der fehlenden Kommunikation und Rezeption der verschiedenen Ansätze (und der teilweise daran gebundenen Perspektiven bzw. Themen) untereinander. An erster Stelle steht viel zu häufig die ,self-certainty“, nicht die ,action“ - und in diesem Fall ist es keine Folge des gewachsenen Drucks durch die Neoliberalisierung der Hochschulen, sondern die Fortsetzung des Denkens in Denkschulen (hier gleichermaßen angelegt an Fleck wie an Kuhn). ${ }^{14}$

Einige der - großen und bereits langanhaltenden (und sich voraussichtlich fortsetzenden) - Geographien sozialer Krisen erfordern aber dringend ,action“ und machen deutlich, dass die hier angestellten Überlegungen zur Materialität in der Sozialgeographie keinesfalls auf die räumliche und/oder soziale Mikroebene beschränkt sind oder in neuen Formen von Selbstresponsibilisierungen münden dürfen: Die im Zusammenhang mit den globalen Umweltkrisen stehenden so-

\footnotetext{
${ }^{13}$ Auch Frost (2016) betont in ihrer Theorie des (Post-)Humanen den hohen Stellenwert der Dynamisierung, z.B. die Biokultivierung des menschlichen Körpers (siehe Schmitz und Degele, 2010 zur Dynamisierung von Embodiment zu Embodying).

${ }^{14}$ Kritisch-reflexiv sei hier also auch auf meine Denkschulen und das sehr bewusst zusammengestellte Literaturverzeichnis hingewiesen.
} 
zialen, ökologischen und ökonomischen Ungleichheiten, seien sie national, regional oder korpo-real, sind Ungerechtigkeiten - die in teils alltäglichen, teils außergewöhnlichen Überlebenskämpfen münden, ,,ways of living and dying matter!“ (Haraway, 2008:88) - und ,matter“ umfasst hier einmal mehr die semiotische und die materielle Ebene. Die Auflösung der unterschiedlichen ontologischen Zugriffe auf Semiotisches und Materielles, Kultur und Natur, Gesellschaft und Umwelt bietet für die Krise der Sozialgeographie und für Geographien sozialer Krisen neue Perspektiven, um klassische wie neuere Formen sozialer und biologischer Ungleichheiten und Ungerechtigkeiten jenseits von Kulturalisierung oder Naturalisierung als transkörperliche Relationen und intra-aktive Mensch-Umwelt-Beziehungen $\mathrm{zu}$ adressieren. Der Fokus auf den menschlichen Körper ist dabei wichtig, da sich soziale, räumliche und ökologische Ungerechtigkeiten dort stofflich materialisieren. D.h. der Fokus bietet in einem radikal-relationalen, posthumanistischen Denkrahmen konkrete Ansatzpunkte, um die klassische sozialund kulturwissenschaftliche Frage ,wie wird eine biologische Differenz zwischen menschlichen Körpern sozial bedeutsam?“, um die in Zeiten multipler Krisen zunehmend elementar werdende biosoziale Frage ,wie wird eine soziale Differenz körperlich-biologisch wirkmächtig?" zu erweitern.

Im Zusammenbringen von Diskurs, Struktur und materiell-stofflichen Wirkmächtigkeiten wird der Körper des Subjekts weiterhin als sozioökonomisch markiert, kulturell kodiert und diskursiv normiert thematisiert, doch basiert sein Da-Sein auch auf biochemischen und -physischen Stoffwechselprozessen. Wenn das epistemologische und ontologische Verständnis vom verkörperten Subjekt als Effekt gesellschaftlicher Inkorporierungs- und diskursiver Verkörperungsprozesse um die stofflich-materielle Somatisierung der räumlichen Umwelt erweitert wird, dann steht weiterhin die Inkorporierung des Gesellschaftlichen - und des Räumlichen iSv Umgebung und Umwelt als Teil des Gesellschaftlichen - im Mittelpunkt, allerdings jenseits der Oberflächen des Repräsentationellen. Sozialgeographisch ist und bleibt der Körper der Ort, an dem sich soziale Strukturen und Diskurse materialisieren - aber auch der Ort, in dem Umweltphänomene (re-)agieren und sich somatisieren.

Datenverfügbarkeit. Für diesen Artikel wurden keine Datensätze genutzt.

Interessenkonflikt. Die Autorin erklärt, dass kein Interessenkonflikt besteht.

Begutachtung. This paper was edited by Benedikt Korf and reviewed by two anonymous referees.
Literatur

Alaimo, S.: Bodily Natures: Science, Environment, and the Material Self, Indiana University Press, Bloomington, 2010.

Alaimo, S.: Exposed: Environmental Politics and Pleasures in Posthuman Times, University of Minnesota Press, Minneapolis, 2016.

Alaimo, S. and Hekman, S. (Eds.): Material Feminisms, Indiana University Press, Bloomington, 2008.

Barad, K.: Posthumanist Performativity: Toward an Understanding of How Matter Comes to Matter, Signs, 28, 801-831, https://doi.org/10.1086/345321, 2003.

Barad, K.: Meeting the Universe Halfway: Quantum Physics and the Entanglement of Matter and Meaning, Duke University Press, Durham, 2007.

Barad, K.: Agentieller Realismus, Suhrkamp, Berlin, 2012.

Barad, K.: Verschränkungen, Merve, Berlin, 2015.

Bauriedl, S., Schier, M. und Strüver, A. (Eds.): Geschlechterverhältnisse, Raumstrukturen, Ortsbeziehungen: Erkundungen von Vielfalt und Differenz im Spatial Turn, Westfälisches Dampfboot, Münster, 2010.

Becker, S. and Otto, A.: Editorial: Natur, Gesellschaft, Materialität: aktuelle Herausforderungen der Politischen Ökologie, Geogr. Helv., 71, 221-227, https://doi.org/10.5194/gh-71-221-2016, 2016.

Bennett, J.: Edible Matter, New Left Review, 45, 133-145, 2007.

Bolte, G.: Epidemiologische Methoden und Erkenntnisse als eine Grundlage für Stadtplanung und gesundheitsfördernde Stadtentwicklung, in: Planung für Gesundheitsfördernde Städte, Herausgebr: Baumgart, S., Köckler, H., Ritzinger, A. und Rüdiger, A., Forschungsberichte der Akademie für Raumforschung und Landesplanung (ARL), 118-134, 2018.

Bourdieu, P.: Rede und Antwort, Suhrkamp, Frankfurt am Main, 1992.

Brown, T.: Andrews, G., Cummins, S., Greenhough, B., Lewis, D., and Power, A.: Health Geographies: A Critical Introduction, Wiley-Blackwell, Oxford, 2017.

Bruckner, H.: Beyond happy meat: Body mapping (dis)connections to animals in alternative food networks, Area, 50, 322-330, https://doi.org/10.1111/area.12381, 2018

Bullard, R. D.: Dumping in Dixie: Race, Class, and Environmental Quality, Westview Press, New York, 2000.

Butler, J.: Bodies that Matter: On the Discursive Limits of "sex", Routledge, London, 1993.

Butler, J.: Hass spricht. Zur Politik des Performativen, Berlin Verlag, Berlin, 1998.

Butler, J.: Die Macht der Geschlechternormen und die Grenzen des Menschlichen, Suhrkamp, Frankfurt am Main, 2009.

Braidotti, R.: Posthumanismus. Leben jenseits des Menschen, Campus, Frankfurt am Main, 2014.

Cağlar, G., Varela, M. C. und Schwenken, H. (Eds.): Geschlecht Macht - Klima: Feministische Perspektiven auf Klima, gesellschaftliche Naturverhältnisse und Gerechtigkeit, Barbara Budrich, Opladen, 2012.

Dörfler, T. and Rothfuß, E.: Lebenswelt, Leiblichkeit und Resonanz: Eine raumphänomenologisch-rekonstruktive Perspektive auf Geographien der Alltäglichkeit, Geogr. Helv., 73, 95-107, https://doi.org/10.5194/gh-73-95-2018, 2018. 
Dzudzek, I. and Strüver, A.: Editorial Subjectivities in crisis, Geogr. Helv., 68, 145-152, https://doi.org/10.5194/gh-68-145-2013, 2013.

Evans, B. and Colls, R.: Making space for fat bodies? A critical account of 'the obesogenic environment', PiHG, 38, 733-753, https://doi.org/10.1177/0309132513500373, 2014.

Evans, A. and Miele, M.: Between Food and Flesh: How Animals are Made to Matter (and Not Matter) within Food Consumption Practices, Environment and Planning D: Society and Space, 30, 298-314, https://doi.org/10.1068/d12810, 2012.

Fausto-Sterling, A.: Sex/Gender: Biology in a Social World, Routledge, New York, 2012.

Foucault, M.: Der Wille zum Wissen: Sexualität und Wahrheit I, Suhrkamp, Frankfurt am Main, 1977.

Foucault, M.: In Verteidigung der Gesellschaft. Vorlesungen am Collège de France (1975-76), Suhrkamp, Frankfurt am Main, 1999.

Foucault, M.: Die Geburt der Biopolitik. Geschichte der Gouvernementalität II, Suhrkamp, Frankfurt am Main, 2006.

Fraser, N.: Scales of Justice: Reimagining Political Space in a Globalizing World, Columbia University Press, New York, 2009.

Frost, S.: Biocultural Creatures: Toward A New Theory of the Human, Duke UP, Durham, 2016.

Gatrell, A. and Elliott, S. (Eds.): Geographies of Health, WileyBlackwell, Oxford, 2015.

Gravlee, C.: How race becomes biology: Embodiment of social inequality, Am. J. Phys. Anthropol., 139, 47-57, https://doi.org/10.1002/ajpa.20983, 2009.

Guthman, J.: Opening the black box of the body in geographical critical obesity research: toward a critical political ecology of fat, Ann. Am. Assoc. Geogr., 102, 951-957, https://doi.org/10.1080/00045608.2012.659635, 2012.

Guthman, J.: Too much food and too little sidewalks? Problematizing the obesogenic environment thesis, Environ. Plan. A, 45, 142-158, https://doi.org/10.1068/a45130, 2013.

Guthman, J.: Doing Justice to Bodies? Reflections on Food Justice, Race, and Biology, Antipode, 46, 1153-1171, https://doi.org/10.1111/j.1467-8330.2012.01017.x, 2014.

Guthman, J.: Binging and purging: agrofood capitalism and the body as socioecological fix, Environ. Plan. A, 47, 2522-2536, https://doi.org/10.1068/a140005p, 2015.

Guthman, J. and DuPuis, M.: Embodying Neoliberalism: Economy, Culture and Politics of Fat, Environ. Plan. D, 24, 427-448, https://doi.org/10.1177/0309132512463258, 2006.

Guthman, J. and Mansfield, B.: The implications of environmental epigenetics: A new direction for geographic inquiry on health, space, and nature-society relations, PiHG, 37, 486-504, https://doi.org/10.1177/0309132512463258, 2013.

Haraway, D.: Die Neuerfindung der Natur, Campus, Frankfurt am Main, 1995.

Haraway, D.: How like a Leaf. An Interview with Thyrza Nichols Goodeve, Routledge, New York, 2000.

Haraway, D.: When Species Meet, University of Minnesota Press, Minneapolis, 2008.

Haraway, D.: Staying with the Trouble: Making Kin in the Chthulucene, Duke University Press, Durham, 2016.

Hasse, J.: Die Abwesenheit der Phänomenologie in der deutschen Humangeographie, Geogr. Helv., 72, 351-360, https://doi.org/10.5194/gh-72-351-2017, 2017.
Hayes-Conroy, J. and Hayes-Conroy, A.: Political ecology of the body: A visceral approach, in: The International Handbook of Political Ecology, edited by: Bryant, R., Edward Elgar, Cheltenham, 659-672, 2015.

Hirth, S.: Ernährung, in: Handbuch kritische Stadtgeographie, Herausgeber: Belina, B., Naumann, M. und Strüver, A., Westfälisches Dampfboot, Münster, 274-279, 2018.

Hochschild, A. R.: Strangers in their Own Land, The New Press, New York, 2016.

Jones III, J. P., Woodward, K., and Marston, S.: Situating Flatness, Trans. Inst. Br. Geogr., 32, 264-276, https://doi.org/10.1111/j.1475-5661.2007.00254.x, 2007.

Kazig, R. und Weichhart, P.: Die Neuthematisierung der materiellen Welt in der Humangeographie, Ber. z. dt. Landeskunde, 83, 109128, 2009.

Krieger, N.: Epidemiology and the People's Health, Oxford UP, Oxford, 2013.

Kuzawa, C. W. and Sweet, E.: Epigenetics and the embodiment of race: Developmental origins of US racial disparities in cardiovascular health, Am. J. Hum. Biol., 21, 2-15, https://doi.org/10.1002/ajhb.20822, 2009.

Landecker, H.: Food as Exposure: Nutritional Epigenetics and the New Metabolism, BioSocieties, 6, 167-194, https://doi.org/10.1057/biosoc.2011.1, 2011.

Löw, C., Volk, K., Leicht, I. und Meisterhans, N. (Eds.): Material Turn: Feministische Perspektiven auf Materialität und Materialismus, Barbara Budrich, Opladen, 2017.

Mansfield, B.: Health as a Nature-Society Question, Environ. Plan. A, 40, 1015-1019, https://doi.org/10.1068/a40366, 2008.

Mansfield, B.: Race and the new epigenetic biopolitics of environmental health, BioSocieties, 7, 352-372, https://doi.org/10.1057/biosoc.2012.22, 2012.

Mansfield, B.: Folded Futurity: Epigenetic Plasticity, Temporality, and New Thresholds of Fetal Life, Sci. Cult., 26, 355-379, https://doi.org/10.1080/09505431.2017.1294575, 2017.

Mansfield, B. and Guthman, J.: Epigenetic life: biological plasticity, abnormality, and new configurations of race and reproduction, Cult. Geogr., 22, 3-20, https://doi.org/10.1177/1474474014555659, 2015.

Marquardt, N. and Strüver, A.: Körper. Machtgeladene Intraaktionen zwischen Biologischem und Sozialem, in: Raumproduktionen II. Theoretische Kontroversen und politische Auseinandersetzungen, Herausgeber: Vogelpohl, A., Michel, B., Lebuhn, H., Hoerning, J. und Belina, B., Westfälisches Dampfboot, Münster, 38-59, 2018.

Marston, S.: The social construction of scale, PiHG, 24, 219-242, https://doi.org/10.1191/030913200674086272, 2000.

Marston, S., Jones III, J. P., and Woodward, K.: Human geography without scale, Trans. Inst. Br. Geogr., 30, 416-432, https://doi.org/10.1111/j.1475-5661.2005.00180.x, 2005.

Meloni, M: Political Biology: Science and Social Values in Human Heredity from Eugenics to Epigenetics, Palgrave Macmillan, London, 2016.

Mol, A.: I eat an apple. On theorising subjectivities, Subjectivity, 22, 28-37, 2008.

Müller, R.: Der epigenetische Körper. Zwischen biosozialer Komplexität und Umweltdeterminismus, Open Gender Journal, https://doi.org/10.17169/ogj, 2017. 
Oßenbrügge, J. und Strüver, A.: Gesundheit, in: Handbuch kritische Stadtgeographie, Herausgeber: Belina, B., Naumann, M. und Strüver, A., Westfälisches Dampfboot, Münster, 290-296, 2018.

Probyn, E.: The spatial imperative of subjectivity, in: Handbook of Cultural Geography, edited by: Anderson, K., Domosh, M., Pile, S., and Thrift, N., Sage Publications, London, 290-299, 2003.

Pulido, L.: Flint, Environmental Racism, and Racial Capitalism, Capitalism Nat. Social., 27, 1-16, https://doi.org/10.1080/10455752.2016.1213013, 2016.

Romero, A. M., Guthman, J., Galt, R. E., Huber, M., Mansfield, B., and Sawyer, S.: Chemical Geographies, GeoHumanities, 3, 158177, https://doi.org/10.1080/2373566X.2017.1298972, 2017.

Runkel, S. and Everts, J.: Geographien sozialer Krisen/Krisen sozialer Geographien, Geogr. Helv., 72, 475-482, https://doi.org/10.5194/gh-72-475-2017, 2017.

Schmitz, S. und Degele, N.: Embodying - ein dynamischer Ansatz für Körper und Geschlecht in Bewegung, in: Gemndered Bodies in Motion, Herausgeber: Degele, N., Schmitz, S., Mangelsdorf, M. und Gramespacher, E., Budrich UNiPress, Opladen, 13-38, 2010.

Schorb, F.: Dick, doof und arm, Droemer, München, 2009.

Schorb, F.: Die Adipositas-Epidemie als politisches Problem. Gesellschaftliche Wahrnehmung und staatliche Intervention, Springer VS, Wiesbaden, 2015.

Sexton, A. E., Hayes-Conroy, A., Sweet, E. L., Miele, M., and Ash, E.: Better than text? Critical reflections on the practices of visceral methodologies in human geography, Geoforum, 82, 200-201, https://doi.org/10.1016/j.geoforum.2017.03.014, 2017.
Taylor, D.: Toxic Communities: Environmental Racism, Industrial Pollution, and Residential Mobility, New York University Press, New York, 2014.

Thrift, N.: Non-representational Theory: Space, Politics, Affect, London, Routledge, 2007.

Tolia-Kelly, D.: The Geographies of Cultural Geography III: Material Geographies, Vibrant Matters and Risking Surface Geographies, PiHG, 37, 153-160, https://doi.org/10.1177/0309132512439154, 2013.

Townshend, T. und Lake, A.: Obesogenic environments: current evidence of the built and food environments, Perspect. Public Health, 137, 38-44, https://doi.org/10.1177/1757913916679860, 2017.

Tuana, N.: Viscous Porosity: Witnessing Katrina, in: Material Feminisms, edited by: Alaimo, S. and Hekman, S., Indiana University Press, Bloomington, 188-213, 2008.

Walker, G.: Beyond Distribution and Proximity: Exploring the Multiple Spatialities of Environmental Justice, Antipode 41, 614 636, https://doi.org/10.1111/j.1467-8330.2009.00691.x, 2009.

Wastl-Walter, D.: Gender Geographien. Geschlecht und Raum als soziale Konstruktionen, Sozialgeographie Kompakt Band 2, Franz Steiner Verlag, Stuttgart, 2010.

Weichhart, P.: Entwicklungslinien der Sozialgeographie, Von Hans Bobek bis Benno Werlen, Franz Steiner Verlag, Stuttgart, 2018.

Werlen, B.: Sozialgeographie. Eine Einführung, Haupt, Bern, 2008.

Young, I. M.: Justice \& the Politics of Difference, Princeton University Press, Princeton, 2011.

Zimring, C.: Clean and White: A History of Environmental Racism in the United States, New York University Press, New York, 2016. 